\title{
First-principles Simulation of Piezoresistivity of Transition Metal Dichalcogenide Monolayers
}

\author{
Koichi Nakamura ${ }^{1,2^{*}}$ \\ ${ }^{1}$ Center for the Promotion of Interdisciplinary Education and Research, Kyoto University, \\ Kyoto 615-8540, Japan \\ ${ }^{2}$ Department of Materials Science and Engineering, Egypt-Japan University of Science and Technology, \\ New Borg El-Arab, Alexandria 21934, Egypt
}

(Received April 5, 2018; accepted July 23, 2018)

Keywords: transition metal dichalcogenide, gauge factor, piezoresistivity, first-principles calculation

Longitudinal gauge factors of transition metal dichalcogenide (TMDC) monolayer models have been evaluated by first-principles calculation. TMDC monolayers have a multivalley/ multipeak electronic structure, and uniaxial tensile strain causes a significant change in electrical conductivity through carrier redistribution in conduction-band valleys or valenceband peaks for some species of doped TMDC monolayers. A high piezoresistivity was observed selectively depending on the combination of transition metal and chalcogen atoms. In this simulation, a p-doped $\mathrm{MoS}_{2}$ monolayer model gave high longitudinal gauge factors of 94.2 and 87.4 at $20^{\circ} \mathrm{C}$ in the carrier concentration range of $10^{17}-10^{19} \mathrm{~cm}^{-3}$.

\section{Introduction}

The exploration of novel high-sensitivity piezoresistive materials is indispensable for expanding the usability and operability of force-sensing micro/nano-electromechanical system (MEMS/NEMS) devices. Transition metal dichalcogenide (TMDC) layers have attracted much attention as new candidates of piezoresistive materials composing MEMS mechanical sensors. In particular, TMDC monolayers containing Group VI transition metals, such as molybdenum (Mo) and tungsten (W), have been the subject of a great deal of interest as a post-graphene twodimensional nanomaterial for application to MEMS devices in various fields of electronics, photonics, and spintronics. ${ }^{(1-10)}$

In contrast to graphene, the TMDC is a typical semiconducting material with a clear band gap, and the effective masses of carrier electrons and holes are nonzero. ${ }^{(11-24)}$ From the viewpoint of piezoresistivity, the measurement or evaluation of gauge factors (GFs) of TMDCs is a key issue for future application to mechanical sensors. Recently, the evaluation of GFs of Mo disulfide $\left(\mathrm{MoS}_{2}\right)$ with 1-3 layers has been reported by the finite-element method with AFM measurement data. ${ }^{(25)}$ On the other hand, the author has already developed the theory and simulation method to evaluate the piezoresistivity of any semiconducting materials on the basis of first-principles calculation. ${ }^{(26-28)}$ The electronic structures of TMDC monolayers have *Corresponding author: e-mail: koichi@cpier.kyoto-u.ac.jp https://doi.org/10.18494/SAM.2018.1957 
been investigated by many researchers, and the author has also presented the details of band structures of TMDC monolayers for the simulation of thermoelectric properties. ${ }^{(11)}$ It is well known that the electronic structures of TMDC monolayers containing Group VI transition metals are affected significantly by spin-orbit coupling. ${ }^{(11-24)}$ In the analysis of TMDC properties regarding stress or strain such as piezoresistivity, the influence of spin-orbit coupling on stress or strain should be sufficiently incorporated.

In this study, longitudinal GFs in doped TMDC monolayer models $M X_{2}(M=\mathrm{Mo}$ and $\mathrm{W} ; X=\mathrm{S}$, $\mathrm{Se}$, and $\mathrm{Te}$ ) have been simulated in terms of first-principles band structures, and the potential of TMDC monolayers for piezoresistive materials shall be discussed.

\section{Details of Simulation Method}

\subsection{First-principles calculation}

The electronic band structures of the TMDC monolayer models have been calculated using the VASP program package ${ }^{(29,30)}$ based on the density functional theory (DFT). ${ }^{(31)}$ For DFT exchange-correlation interaction, the generalized-gradient approximation method was used with the Perdew-Wang (PW91) functional. ${ }^{(32,33)}$ The projector augmented wave (PAW) method was adopted, ${ }^{(34)}$ and the cutoff energy for wave functions of electrons was set at 40 Ry $(544 \mathrm{eV})$. Spin-orbit coupling was included in all DFT calculations. The $k$-point sampling was set by the Monkhorst-Pack grids ${ }^{(35)}$ of $6 \times 6 \times 1$ for geometry optimization and of $48 \times 48 \times 1$ for carrier integration in the Brillouin zone.

Figure 1 shows unit cells of the TMDC monolayer models in this study. The configuration of Mo and $\mathrm{W}$ atoms is trigonal prismatic for $\mathrm{Mo} X_{2}$ and $\mathrm{W} X_{2}$. For the direction vertical to

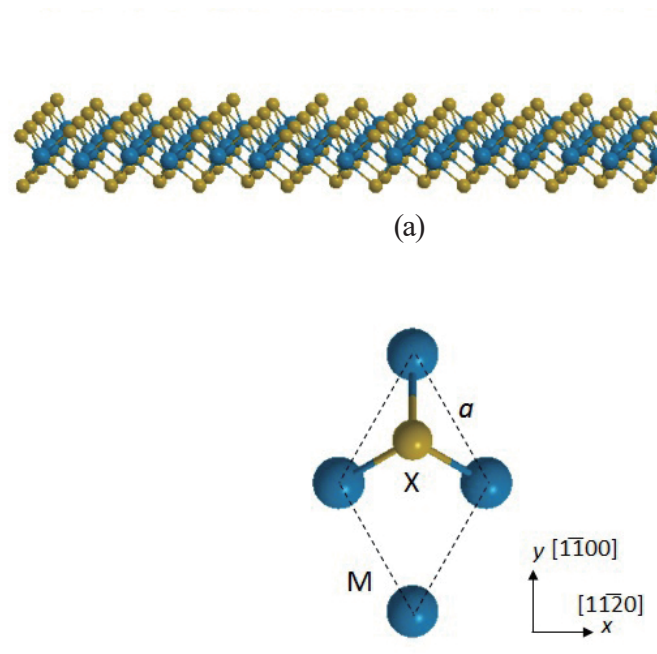

(b)

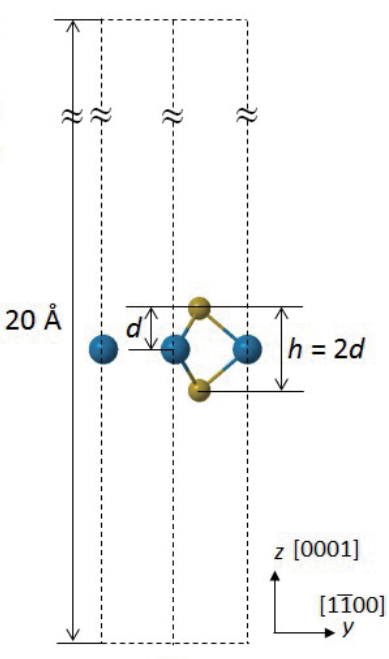

(c)

Fig. 1. (Color online) TMDC models for first-principles calculation ( $M=\mathrm{Mo}$ and $\mathrm{W} ; X=\mathrm{S}, \mathrm{Se}$, and $\mathrm{Te}$ ): (a) image of TMDC monolayer and (b) top and (c) side views of unit cell of TMDC monolayer model. 
the two-dimensional monolayer, the vacuum space was introduced for establishing a threedimensional periodic boundary condition by setting the fixed vertical cell parameter to $20 \AA$. Table 1 shows the optimized geometrical parameters $a$ and $d$ shown in Fig. 1 for the TMDC monolayer models, ${ }^{(11)}$ and the volume per unit cell was defined by $V=(\sqrt{3} / 2) a^{2} h$, where $h$ is the layer thickness and is conveniently regarded as $2 d$ in this study.

The effect of uniaxial tensile strain on the TMDC plane was represented by partial optimization with a fixed scale along the tensile direction. In this study, $1 \%$ tensile-strained models were introduced by applying the uniaxial tensile strain $\varepsilon=0.01$ along the [1120] and [1100] directions. As reported by Yu et al., ${ }^{(36)}$ Poisson's ratios were obtained as negative values, ranging from -0.02 to -0.10 , for all TMDC monolayer models.

\subsection{Evaluation of gauge factor}

In the two-dimensional transport model of doped semiconductors with a multivalley and/or multipeak electronic structure, the electrical conductivity tensor $\boldsymbol{G}$ or electrical resistivity tensor $\boldsymbol{\rho}$ can be represented as a $2 \times 2$ tensor in terms of carrier densities and effective mass tensors by

$$
\boldsymbol{G}=(\boldsymbol{\rho})^{-1}=e^{2}\left[\sum_{j \in \mathrm{CB} \alpha} \sum_{\alpha} n_{j, \alpha}\left(\boldsymbol{m}_{j, \alpha}^{*}\right)^{-1} \tau_{j, \alpha}+\sum_{j \in \mathrm{VB}} \sum_{\beta} n_{j, \beta}\left(\boldsymbol{m}_{j, \beta}^{*}\right)^{-1} \tau_{j, \beta}\right],
$$

where $n_{j, \alpha}$ is the $j$ th conduction-band (CB) carrier electron density for the $\mathrm{CB}$ valley $\alpha, p_{j, \beta}$ is the $j$ th valence-band (VB) hole density for the VB peak $\beta, \boldsymbol{m}_{j, \alpha}^{*}$, and $\boldsymbol{m}_{j, \beta}^{*}$ are band effective mass tensors, $\boldsymbol{\tau}_{j, \alpha}$ and $\boldsymbol{\tau}_{j, \beta}$ are relaxation time tensors, and $e^{2}$ is the square of the absolute value of the elementary electric charge. ${ }^{(37)}$ An excess or lack of electrons as large as one particle per unit cell, in order to carry out regular first-principles calculation in the n- or p-doped semiconductor state, should lead to an enormous overestimation of carrier concentration. In actual doped TMDC monolayers, the total number of carrier electrons or holes per unit cell, $\delta$, must be less by a few orders than 1 . Under the condition that a small amount of carrier occupation causes no significant changes in band structure, $\delta$ can be given by an appropriate shift in the Fermi energy $E_{F}$ as

Table 1

Optimized geometrical parameters for TMDC monolayer models.

\begin{tabular}{llllll}
\hline \multirow{2}{*}{$X$} & \multicolumn{2}{c}{$\mathrm{Mo} X_{2}$} & & \multicolumn{2}{c}{$\mathrm{W} X_{2}$} \\
\cline { 2 - 3 } \cline { 5 - 6 } & $a(\AA)$ & & & $a(\AA)$ & $d(\AA)$ \\
\hline Disulfide (S) & 3.190 & 1.561 & & 3.190 & 1.563 \\
Diselenide (Se) & 3.311 & 1.690 & & 3.312 & 1.695 \\
Ditelluride (Te) & 3.580 & 1.803 & & 3.580 & 1.803 \\
\hline
\end{tabular}




$$
\begin{gathered}
\delta=\sum_{j \in \mathrm{CB}} \sum_{\alpha} n_{j, \alpha} V=\sum_{j \in \mathrm{CB}} \sum_{\boldsymbol{k}} w_{\boldsymbol{k}}\left[\exp \left(\frac{E_{j, \boldsymbol{k}}-E_{F}}{k_{B} T}\right)+1\right]^{-1}, \\
\delta=\sum_{j \in \mathrm{VB} \beta} \sum_{\beta} p_{j, \beta} V=\sum_{j \in \mathrm{VB}} \sum_{\boldsymbol{k}} w_{\boldsymbol{k}}\left[\exp \left(-\frac{E_{j, \boldsymbol{k}}-E_{F}}{k_{B} T}\right)+1\right]^{-1},
\end{gathered}
$$

in the n- or p-type carrier occupation with the temperature $T$, respectively, where $E_{j, k}$ is the intrinsic-semiconductor-state band energy of the $j$ th subband at the $\boldsymbol{k}$ point, $w_{\boldsymbol{k}}$ is the $k$-point weight for $\boldsymbol{k}$, and $k_{B}$ is the Boltzmann constant. Practically, $\delta=N V$ was set to an appropriate constant that corresponds to the carrier concentration $N$ ranging from $1 \times 10^{17}$ to $1 \times 10^{19} \mathrm{~cm}^{-3}$, and then $E_{F}$ was solved according to Eq. (2) or (3). The values of $n_{j, \alpha}$ and $p_{j, \beta}$ were respectively determined by regional partitioning as

$$
\begin{gathered}
n_{j, \alpha}=\sum_{\boldsymbol{k} \in R_{\alpha}} w_{\boldsymbol{k}}\left[\exp \left(\frac{E_{j, \boldsymbol{k}}-E_{F}}{k_{B} T}\right)+1\right]^{-1} / V, \\
p_{j, \beta}=\sum_{\boldsymbol{k} \in R_{\beta}} w_{\boldsymbol{k}}\left[\exp \left(-\frac{E_{j, \boldsymbol{k}}-E_{F}}{k_{B} T}\right)+1\right]^{-1} / V,
\end{gathered}
$$

where $R_{\alpha}$ is the region of the CB valley $\alpha$ and $R_{\beta}$ is the region of the VB peak $\beta .^{(26)}$

The band effective mass tensors in each region can be defined as $2 \times 2$ tensors,

$$
\left(\boldsymbol{m}_{j, \gamma}^{*}\right)^{-1}= \pm \frac{1}{\hbar^{2}} \boldsymbol{R}\left(\begin{array}{cc}
\partial^{2} E_{j} / \partial k_{x}^{2} & \partial^{2} E_{j} / \partial k_{x} \partial k_{y} \\
\partial^{2} E_{j} / \partial k_{y} \partial k_{x} & \partial^{2} E_{j} / \partial k_{y}^{2}
\end{array}\right) \boldsymbol{R}^{\mathrm{T}}
$$

at the minimum band energy $E_{j}$ in $R_{\alpha}$ or the maximum $E_{j}$ in $R_{\beta}$. $\hbar$ is equal to Planck's constant divided by $2 \pi$ and $\boldsymbol{R}$ is the planar rotation matrix around the $k_{z} \|$ [0001] axis. On the righthand side in Eq. (6), a positive sign is adopted for carrier electrons $(\gamma=\alpha)$ and a negative sign for holes $(\gamma=\beta)$. In this paper, the $x$ - and $y$-directions can be respectively defined as the [11 $\overline{2} 0]$ and [1 $1 \overline{1} 00$ ] directions, and $\boldsymbol{R}$ becomes a unit matrix in this definition. For the relaxation times, we have adopted the approximation that all of the band relaxation times are equal and constant regardless of strain, ${ }^{(26-28)}$ because the variation rate of carrier conductivity can be easily and adequately represented by cancelling most of the band relaxation times.

Under the uniaxial tensile strain $\varepsilon$, the longitudinal GF $K_{l}$ is defined as

$$
K_{l}=\Delta \rho_{l} / \rho_{l}^{0} \varepsilon,
$$


where $\rho_{l}^{0}$ is the longitudinal diagonal element of the resistivity tensor $\boldsymbol{\rho}$ without strain and $\Delta \rho_{l}$ is the variation in resistivity in the direction toward the uniaxial tensile strain.

\section{Results and Discussion}

\subsection{Carrier redistribution by uniaxial strain}

The details of the multivalley/multipeak band structure for the TMDC monolayer models have been reported in the author's previous study. ${ }^{(1)}$ The band diagrams in the vicinities of the CB bottom and VB top for each TMDC monolayer model are shown in Fig. 2. For the CB bottom of monolayers, there are deep valleys not only at the $\mathrm{K}$ points but at the $\Gamma \mathrm{K}$ points along the $\Gamma-\mathrm{K}$ paths. Valleys at the $\Gamma \mathrm{M}$ points along the $\Gamma-\mathrm{M}$ paths are actually very shallow for the direction perpendicular to the $\Gamma-\mathrm{M}$ paths toward the $\Gamma \mathrm{K}$ valleys, so the occupation of carrier electrons on the $\Gamma \mathrm{M}$ valleys can be ignored. For the VB top, there are peaks at the $\mathrm{K}$ and $\Gamma$ points. Figure 3 shows the regional partitioning to define $R_{\alpha}\left(R_{\mathrm{K}}, R_{\Gamma \mathrm{K} 1}\right.$, and $\left.R_{\Gamma \mathrm{K} 2}\right)$ and $R_{\beta}\left(R_{\mathrm{K}}\right.$ and $R_{\Gamma}$ ) for computing $n_{j, \alpha}$ and $p_{j, \beta}$ for each valley or peak for Eqs. (4) and (5). Six K valleys and six $\mathrm{K}$ peaks on the corners of the Brillouin zone respectively remain equivalent with the application of any strain, ${ }^{(27)}$ and they can be treated as one valley and one peak. On the other hand, the uniaxial strain gives a small difference in band energy level among six $\Gamma \mathrm{K}$ valleys. By applying the uniaxial strain in the $[11 \overline{2} 0]$ and [1 $\overline{1} 00]$ directions, six $\Gamma \mathrm{K}$ valleys are split into four $\Gamma \mathrm{K}_{1}$ valleys and two $\Gamma \mathrm{K}_{2}$ valleys.

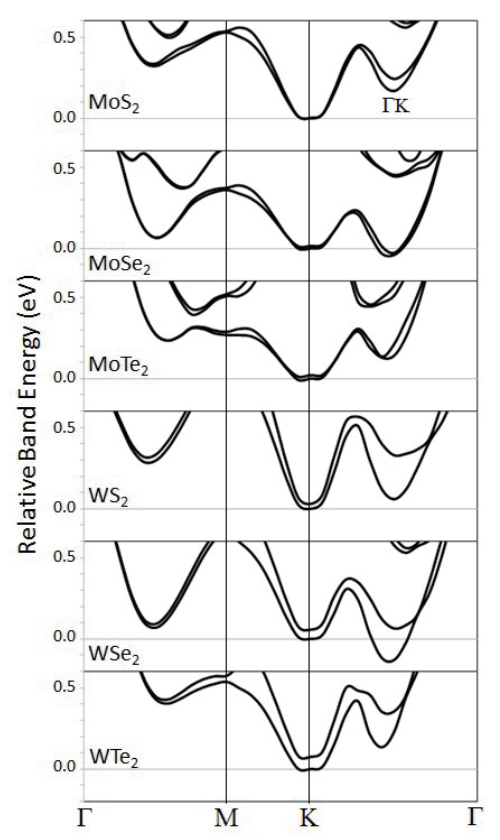

(a)

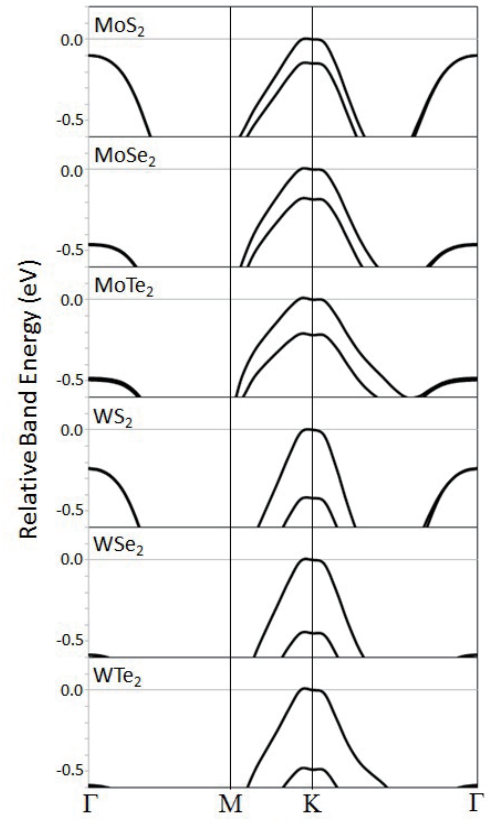

(b)

Fig. 2. Band energy diagrams of the strain-free TMDC monolayer models for (a) CB bottom and (b) VB top. The energy zero is defined by the energy at the $\mathrm{K}$ point for $\mathrm{CB}$ and $\mathrm{VB}$. 


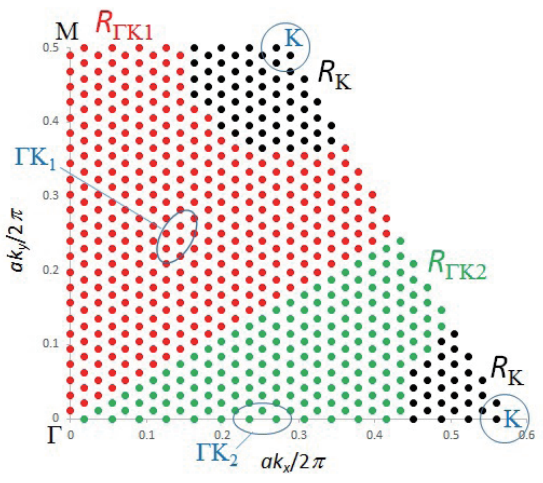

(a)

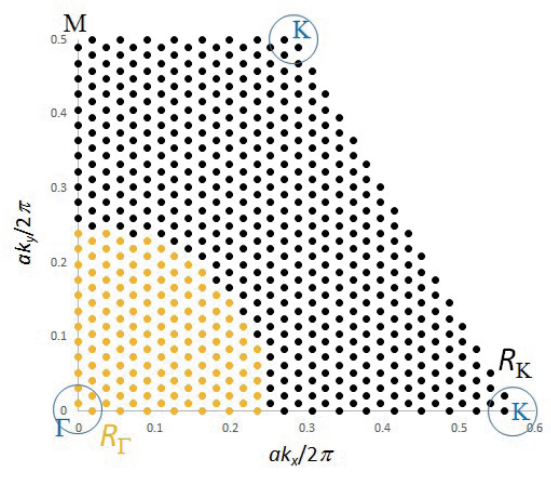

(b)

Fig. 3. (Color online) Regional partitioning of (a) CB bottom and (b) VB top for the range of $k_{x}, k_{y}>0$. Plots are $48 \times 48 \times 1$ Monkhorst-Pack grids for carrier integration .

The temperature dependences of the carrier electron occupation ratios for the strain-free and $[11 \overline{2} 0]$ tensile-strained TMDC monolayer models are shown in Fig. 4. For n-doped $\mathrm{MoSe}_{2}$ and $\mathrm{WSe}_{2}$, carrier electrons are distributed on both the $\Gamma \mathrm{K}$ and $\mathrm{K}$ valleys, because the $\Gamma \mathrm{K}$ valleys in $\mathrm{MoSe}_{2}$ lie on almost the same band energy level of the $\mathrm{K}$ valleys, and the $\Gamma \mathrm{K}$ valleys clearly lie lower than the $\mathrm{K}$ valleys in $\mathrm{WSe}_{2}$ at the calculation level in this paper. By applying the uniaxial strain, a remarkable redistribution of carrier electrons can be easily triggered from the $\Gamma \mathrm{K}$ valleys to the $\mathrm{K}$ valleys in both $\mathrm{n}$-doped $\mathrm{MoSe}_{2}$ and $\mathrm{WSe}_{2}$ monolayer models. On the other hand, the redistribution of carrier electrons can hardly be observed in n-doped $\mathrm{MoS}_{2}, \mathrm{MoTe}_{2}$, $\mathrm{WS}_{2}$, and $\mathrm{WSe}_{2}$. The changes in carrier electron occupation ratio by the tensile strain for $\mathrm{MoSe}_{2}$ and $\mathrm{WSe}_{2}$ models can be supported by the band energy diagram for the corresponding tensilestrained models shown in Fig. 5. The relative band energy levels at the $\Gamma \mathrm{K}_{1}$ and $\Gamma \mathrm{K}_{2}$ valleys to the $\mathrm{K}$ valley are raised by 0.05 and $0.03 \mathrm{eV}$ for $\mathrm{MoSe}_{2}$ and $\mathrm{WSe}_{2}$, respectively, owing to the $1 \%$ tensile strains.

For the VB of monolayer models, all holes are located at the $\mathrm{K}$ point in $\mathrm{p}$-doped TMDCs except for $\mathrm{MoS}_{2}$, and the redistribution of holes cannot occur in the presence of any strain. Only in the $\mathrm{MoS}_{2}$ model, the band energy of the $\Gamma$ peak is close to that of the $\mathrm{K}$ peak, and holes are distributed on both the $\mathrm{K}$ and $\Gamma$ peaks for $\mathrm{p}$-doped $\mathrm{MoS}_{2}$. Figure 6 shows the temperature dependences of the hole occupation ratios for the strain-free and [11 $\overline{2} 0]$ tensilestrained monolayer models. The uniaxial strain can give rise to the redistribution of holes from the $\mathrm{K}$ point to the $\Gamma$ point. The change in hole occupation ratio by the tensile strain for the $\mathrm{MoS}_{2}$ model can also be supported by the band energy diagram for the corresponding tensilestrained models shown in Fig. 7. The difference in band energy level between the $\Gamma$ and $\mathrm{K}$ peaks is clearly reduced by $0.06-0.07 \mathrm{eV}$ for $\mathrm{MoS}_{2}$ owing to the $1 \%$ tensile strains. The carrier occupation ratios shown in Figs. 4 and 6 are almost independent of carrier concentration in the range of $10^{17}-10^{19} \mathrm{~cm}^{-3}$ for all TMDC models. The [1100] tensile strain gives almost the same effect as the $[11 \overline{2} 0]$ tensile strain on the carrier occupation ratio except for the $\Gamma K_{1}$ and $\Gamma K_{2}$ valleys. 


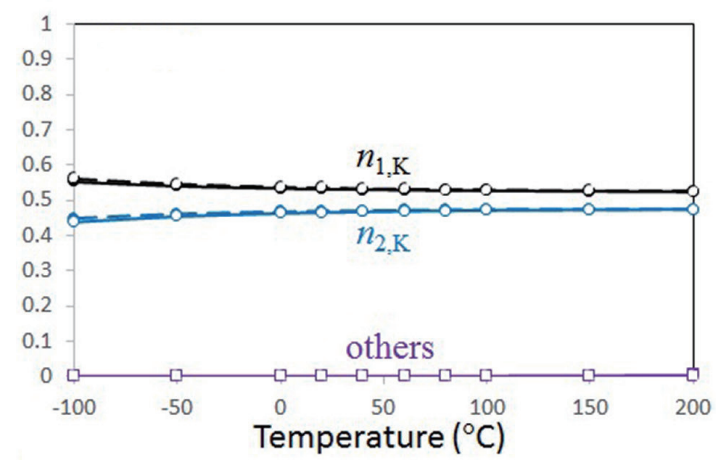

(a)

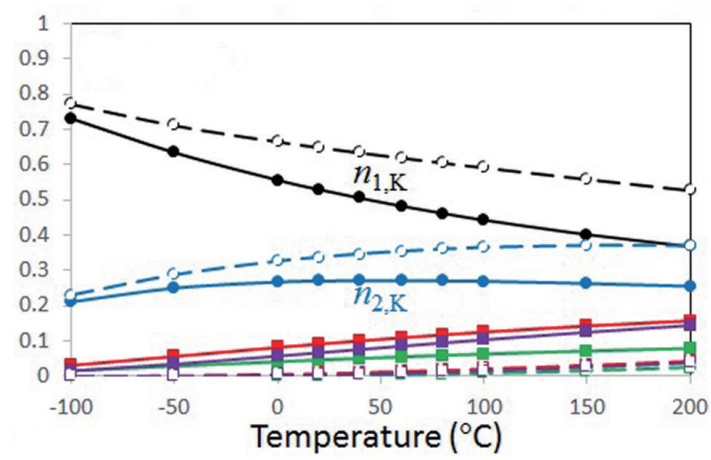

(b)

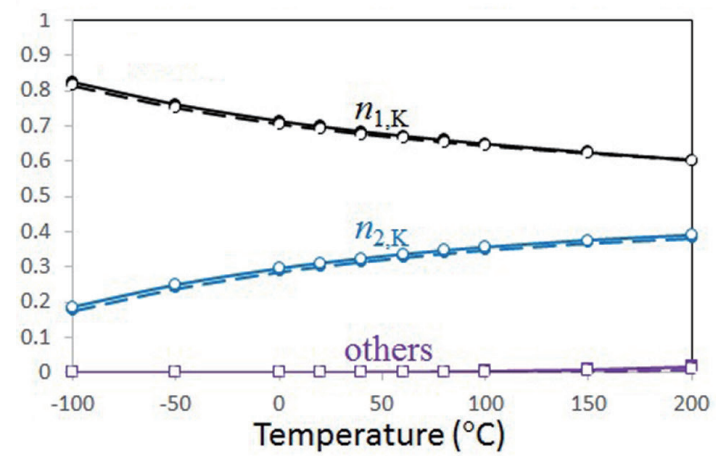

(c)

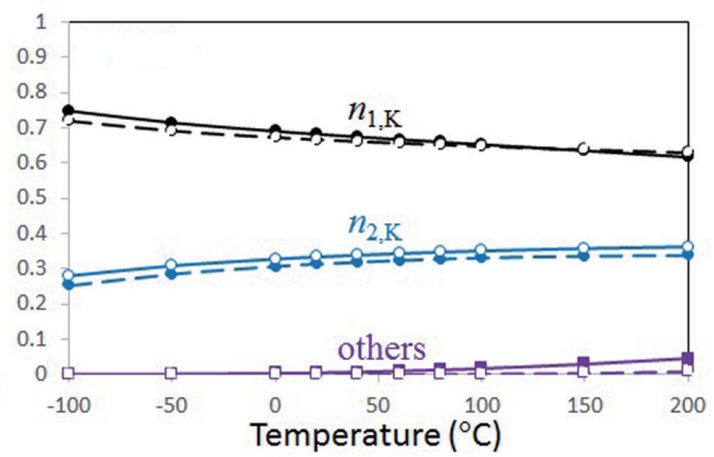

(d)

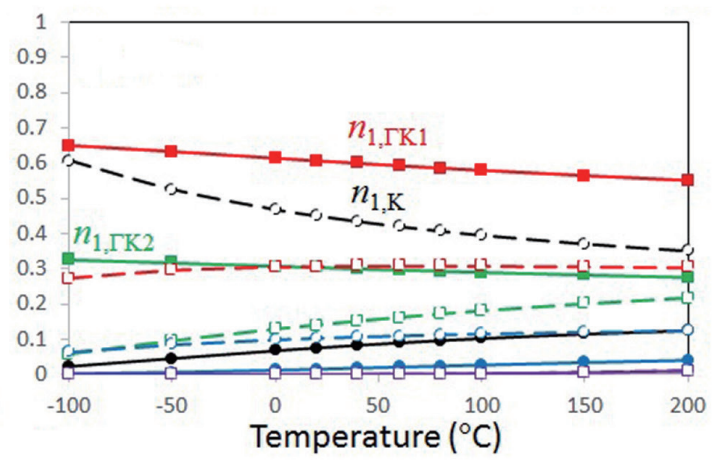

(e)

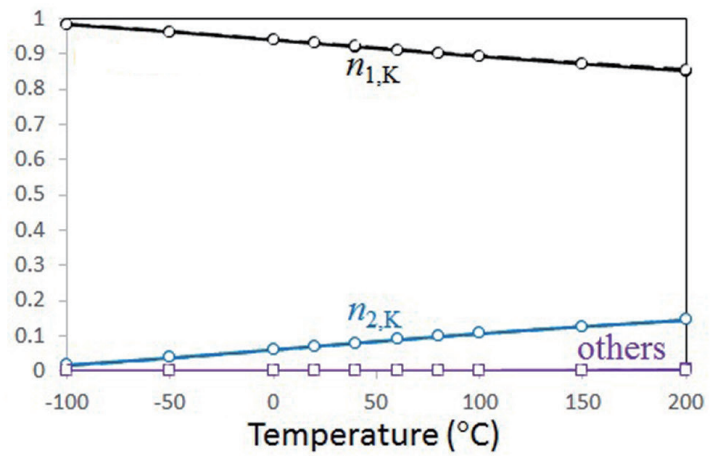

(f)

Fig. 4. (Color online) Temperature dependences of the carrier electron occupation ratios for the n-doped strainfree and $[11 \overline{2} 0]$ tensile-strained TMDC monolayer models at $10^{18} \mathrm{~cm}^{-3}$ carrier concentration: (a) $\mathrm{MoS}_{2}$, (b) $\mathrm{MoSe}_{2}$, (c) $\mathrm{MoTe}_{2}$, (d) $\mathrm{WS}_{2}$, (e) $\mathrm{WSe}_{2}$, and (f) $\mathrm{WTe}_{2}$. The black, blue, red, and green curves correspond to $n_{1, \mathrm{~K}}, n_{2, \mathrm{~K}}, n_{1, \Gamma \mathrm{K} 1}$, and $n_{1, \Gamma K 2}$, respectively, for the $j$ th lowest CB subbands in unit of $\delta / V$. The solid lines and filled marks are for the strainfree models, whereas the dashed line and open marks are for the [11 $\overline{2} 0]$ tensile-strained models.

\subsection{Longitudinal GFs}

The calculated longitudinal GFs of the doped TMDC monolayer models are shown in Table 2. The carrier concentration dependence of GFs was hardly observed in the carrier concentration range of $10^{17}-10^{19} \mathrm{~cm}^{-3}$, and the calculated GFs show a similar tendency between the [11 $\left.\overline{2} 0\right]$ and $[1 \overline{100}]$ directions for all TMDC monolayer models. The effective masses in these TMDC 


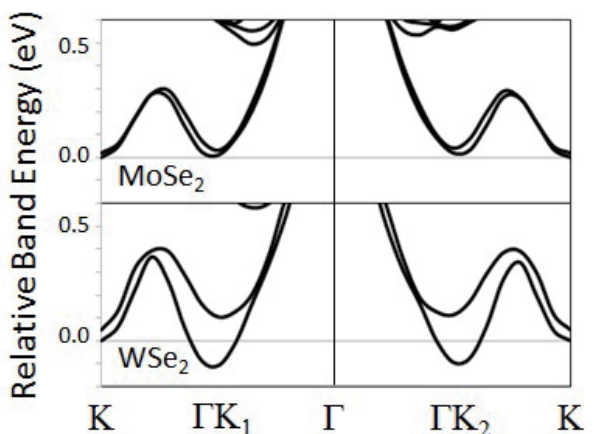

(a)

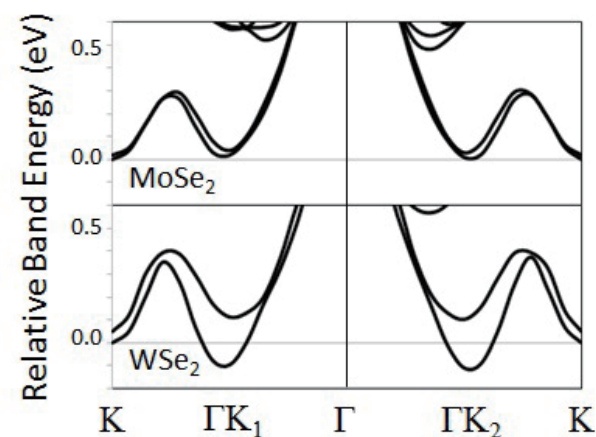

(b)

Fig. 5. Band energy diagrams of the (a) [1 $1 \overline{0} 00]$ and (b) [11 $\overline{2} 0]$ tensile-strained TMDC monolayer models for CB bottom. The energy zero is defined by the energy at the $\mathrm{K}$ point for $\mathrm{CB}$.

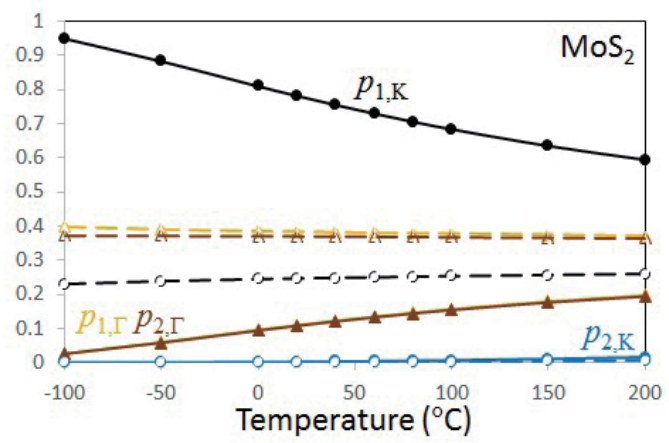

Fig. 6. (Color online) Temperature dependences of the hole occupation ratios for the p-doped strain-free and [1120] tensile-strained $\mathrm{MoS}_{2}$ monolayer models at $10^{18} \mathrm{~cm}^{-3}$ carrier concentration. The black, blue, orange, and brown curves correspond to $p_{1, \mathrm{~K}}, p_{2, \mathrm{~K}}, p_{1, \Gamma}$, and $p_{2, \Gamma}$, respectively, for the $j$ th highest VB subbands in unit of $\delta / V$. The solid lines and filled marks are for the strain-free models, whereas the dashed line and open marks are for the [11 $\overline{2} 0]$ tensile-strained models.

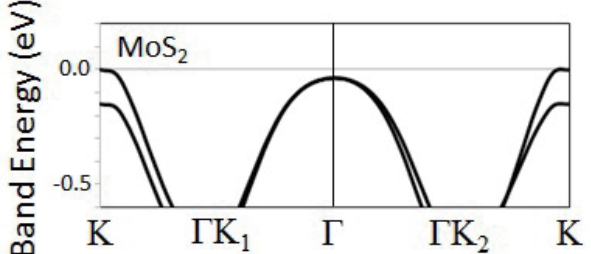

(a)

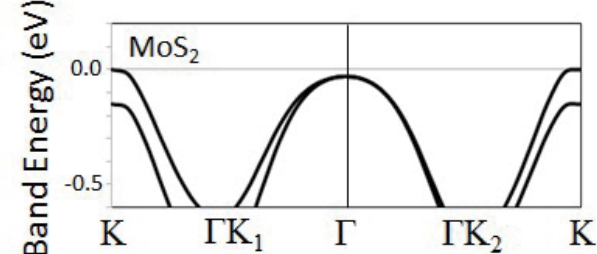

(b)

Fig. 7. Band energy diagrams of the (a) [1 $1 \overline{100}]$ and (b) [11 $\overline{2} 0]$ tensile-strained TMDC monolayer models for VB top. The energy zero is defined by the energy at the K point for VB.

models are hardly affected by uniaxial strain or stress, so the piezoresistivity arises mainly from the carrier redistribution. An effect of the intervalley scattering is small enough to neglect for the models with the effective carrier redistribution. For the models where uniaxial strain or stress does not cause the carrier redistribution, the GFs are very small. As discussed above, the 
Table 2

Calculated longitudinal GFs of TMDC monolayer models at $20^{\circ} \mathrm{C}$.

\begin{tabular}{|c|c|c|c|c|}
\hline \multirow{2}{*}{$\begin{array}{l}\text { TMDC } \\
\text { monolayer }\end{array}$} & \multicolumn{2}{|c|}{ n-doped $\left(10^{18} \mathrm{~cm}^{-3}\right)$} & \multicolumn{2}{|c|}{$\mathrm{p}$-doped $\left(10^{18} \mathrm{~cm}^{-3}\right)$} \\
\hline & {$[11 \overline{2} 0]$ direction } & [1100] direction & [1120] direction & [11 100$]$ direction \\
\hline $\mathrm{MoS}_{2}$ & -0.8 & 0.1 & 94.2 & 87.4 \\
\hline $\mathrm{MoSe}_{2}$ & -8.7 & -5.7 & 2.7 & 3.2 \\
\hline $\mathrm{MoTe}_{2}$ & 0.1 & 0.7 & 2.3 & 2.5 \\
\hline $\mathrm{WS}_{2}$ & -0.1 & 0.8 & 3.4 & 3.8 \\
\hline $\mathrm{WSe}_{2}$ & -20.6 & -16.4 & 2.5 & 2.8 \\
\hline $\mathrm{WTe}_{2}$ & 2.2 & 3.2 & 1.9 & 2.2 \\
\hline
\end{tabular}

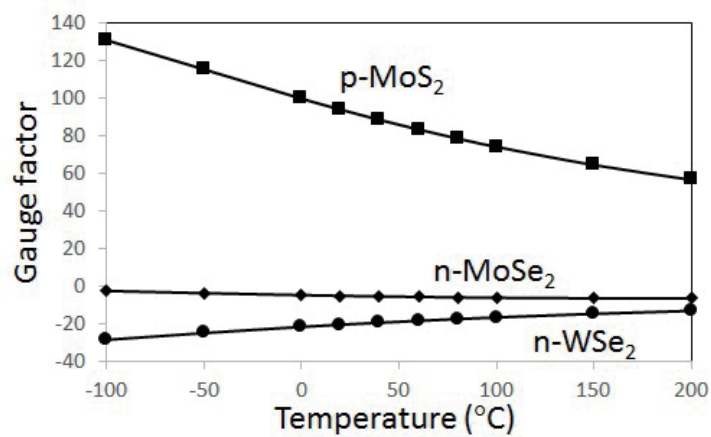

Fig. 8. Temperature dependence of longitudinal GFs of n-MoSe $2, n-\mathrm{WSe}_{2}$, and $\mathrm{p}-\mathrm{MoS}_{2}$ monolayer models in $10^{18} \mathrm{~cm}^{-3}$ carrier concentration. All plots are averaged values of GFs for [1100] and [11 $\left.\overline{2} 0\right]$ directions.

effective carrier redistribution can be observed in n-doped $\mathrm{MoSe}_{2}$ and $\mathrm{WSe}_{2}$ and $\mathrm{p}$-doped $\mathrm{MoS}_{2}$ monolayer models, and then significant GFs are obtained in these models. In particular, the p-doped $\mathrm{MoS}_{2}$ monolayer model gives high longitudinal GFs, namely, 94.2 and 87.4 for the [11 $\left.\overline{2} 0\right]$ and [1ํㅣㅇㅣ directions, respectively.

However, the GFs in n-doped $\mathrm{MoSe}_{2}$ and $\mathrm{WSe}_{2}$ models are not very high, because the effective masses at the $\mathrm{K}$ valleys are not far from those at the $\Gamma \mathrm{K}$ valleys. In contrast, the diagonal elements of the reciprocal effective-mass tensor in Eq. (6) at the $\Gamma$ peaks are $0.21-0.33$ times as much as those at the $\mathrm{K}$ peaks for the $\mathrm{MoS}_{2}$ monolayer model, and therefore, a drastic decrease in electric conductivity should be observed by the hole transfer from the $\mathrm{K}$ peaks to the $\Gamma$ point.

For each of $\mathrm{n}$-doped $\mathrm{MoSe}_{2}$ and $\mathrm{WSe}_{2}$ and $\mathrm{p}$-doped $\mathrm{MoS}_{2}$ monolayer models, the longitudinal

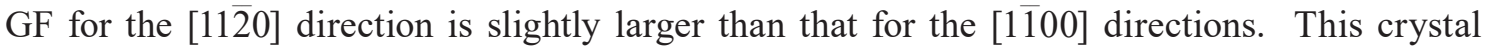
anisotropy in GF is due to a tiny difference in the amount of carrier redistribution in the firstprinciples calculation. As discussed above, the mechanisms of the longitudinal piezoresistance effect in n-doped $\mathrm{MoSe}_{2}$ and $\mathrm{WSe}_{2}$ and p-doped $\mathrm{MoS}_{2}$ monolayer models are common for the [11 20$]$ and [1 $\overline{100}$ ] directions; therefore, it can be regarded that the crystal anisotropy in piezoresistance should be small. The temperature dependence of longitudinal GFs in specific models is shown in Fig. 8. Similarly to general semiconducting materials, the absolute values of longitudinal GFs decrease as temperature increases. 


\section{Conclusions}

The longitudinal GFs of $M X_{2}$ TMDC monolayer models $(M=$ Mo and $\mathrm{W} ; X=\mathrm{S}$, Se, and Te) have been evaluated by first-principles calculation. TMDC monolayers have a multivalley/ multipeak electronic structure, and effective regional partitioning for carrier redistribution has been presented. For some species of doped TMDC monolayers, uniaxial tensile strain causes a significant change in electrical conductivity through carrier redistribution in $\mathrm{CB}$ valleys or VB peaks. A high piezoresistivity was observed selectively depending on the combination of $M$ and $X$. In this simulation, a p-doped $\mathrm{MoS}_{2}$ monolayer model gave high longitudinal GFs of 94.2 for the [1120] direction and 87.4 for the [1 100$]$ direction at $20^{\circ} \mathrm{C}$ in the carrier concentration range of $10^{17}-10^{19} \mathrm{~cm}^{-3}$, based on the hole transfer from the $\mathrm{K}$ peaks to the $\Gamma$ point by the uniaxial strain or stress with the change in effective mass.

\section{Acknowledgments}

This work was partly conducted under the "Project for Egypt-Japan University of Science and Technology (E-JUST) Phase 2" sponsored by the Japan International Cooperation Agency (JICA), for which the author expresses his gratitude.

\section{References}

1 J. A. Wilson and A. D. Yoffe: Adv. Phys. 18 (1963) 193.

2 A. Splendiani, L. Sun, Y. Zhang, T. Li, J. Kim, C.-Y. Chim, G. Galli, and F. Wang: Nano Lett. 10 (2010) 1271.

3 B. Radisavljevic, A. Radenovic, J. Brivio, V. Giacometti, and A. Kis: Nat. Nanotechnol. 6 (2011) 147.

4 H. Zeng, J. Dai, W. Yao, D. Xiao, and X. Cui: Nat. Nanotechnol. 7 (2012) 490.

5 K. F. Mak, K. He, J. Shan, and T. F. Heinz: Nat. Nanotechnol. 7 (2012) 494.

6 Q. H. Wang, K. Kalantar-Zadeh, A. Kis, J. N. Coleman, and M. S. Strano: Nat. Nanotechnol. 7 (2012) 699.

7 Y. Chen, J. Xi, D. O. Dumcenco, Z. Liu, K. Suenaga, D. Wang, Z. Shuai, Y.-S. Huang, and L. Xie: ACS Nano 7 (2013) 4610.

8 R. S. Sundaram, M. Engel, A. Lombardo, R. Krupke, A. C. Ferrari, Ph. Avouris, and M. Steiner: Nano Lett. 13 (2013) 1416.

9 O. Lopez-Sanchez, D. Lembke, M. Kayci, A. Radenovic, and A. Kis: Nat. Nanotechnol. 8 (2013) 497.

10 S. B. Desai, S. R. Madhvapathy, A. B. Sachid, J. P. Llinas, Q. Wang, G. H. Ahn, G. Pitner, M. J. Kim, J. Bokor, C. Hu, H.-S. P. Wong, and A. Javey: Science 354 (2016) 99.

11 K. Nakamura: Jpn. J. Appl. Phys. 57 (2018) 06HE04.

12 L. F. Mattheiss: Phys. Rev. B 8 (1973) 3719.

13 A. Kuc, N. Zibouche, and Y. Heine: Phys. Rev. B 83 (2011) 245213.

14 Z. Y. Zhu, Y. C. Cheng, and U. Schwingenschlögl: Phys. Rev. B 84 (2011) 153402.

15 T. Cao, G. Wang, W. Han, H. Ye, C. Zhu, J. Shi, Q. Niu, P. Tan, E. Wang, B. Liu, and J. Feng: Nat. Commun. 3 (2012) 887.

16 D. Xiao, G.-B. Liu, W. Feng, X. Xu, and W. Yao: Phys. Rev. Lett. 108 (2012) 196802.

17 A. Kumar and P. K. Ahluwalla: Eur. Phys. J. B 85 (2012) 186.

18 A. Ramasubramaniam: Phys. Rev. B 86 (2012)115409.

19 C. Gong, H. Zhang, W. Wang, L. Colobmo, R. M. Wallace, and K. Cho: Appl. Phys. Lett. 103 (2013) 053513.

20 H.-P. Komsa and A. V. Krasheninnikov: Phys. Rev. B 88 (2013) 085318.

21 G.-B. Liu, W.-Y. Shan, Y. Yao, W. Yao, and D. Xiao: Phys. Rev. B 88 (2013) 085433.

22 K. Kośmider, J. W. González, and J. Fernández-Rossier: Phys. Rev. B 88 (2013) 245436.

23 X Xu, W. Yao, D. Xiao, and T. F. Heinz: Nat. Phys. 10 (2014) 343.

24 J. A. Reyes-Retana and F. Cervantes-Sodi: Sci. Rep. 6 (2016) 24093.

25 S. Manzeli, A. Allain, A. Ghadimi, and A. Kis: Nano Lett. 15 (2015) 5330. 
26 K. Nakamura, Y. Isono, T. Toriyama, and S. Sugiyama: Phys. Rev. B 80 (2009) 045205.

27 K. Nakamura, T. Toriyama, and S. Sugiyama: J. Appl. Phys. 50 (2011) 06 GE05.

28 K. Nakamura, Y. Isono, and T. Toriyama: Jpn. J. Appl. Phys. 47 (2008) 5132.

29 G. Kresse and J. Hafner: Phys. Rev. B 47 (1993) R558.

30 G. Kresse and J. Furthmüller: Phys. Rev. B 54 (1996) 11169.

31 P. Hohenberg and W. Kohn: Phys. Rev. 136 (1964) B864.

32 J. P. Perdew and Y. Wang: Phys. Rev. B 45 (1992) 13244.

33 J. P. Perdew, J. A. Chevary, S. H. Vosko, K. A. Jackson, M. R. Pederson, D. J. Singh, and C. Fiolhais: Phys. Rev. B 46 (1992) 6671.

34 P. E. Blöchl: Phys. Rev. B 50 (1994) 17953.

35 H. J. Monkhorst and J. D. Pack: Phys. Rev. B 13 (1976) 5188.

36 L. Yu, Q. Yan, and A. Ruzsinszky: Nat. Commun. 8 (2017) 15224.

37 C. Kittel: Introduction to Solid State Physics (Wiley, New York, 2005) 8th ed., p. 200.

\section{About the Author}

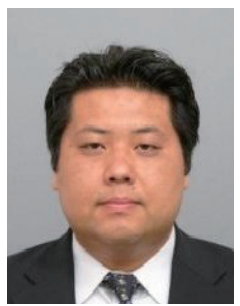

Koichi Nakamura received his B.S., M.S., and Ph.D. degrees in molecular engineering from Kyoto University, Japan in 1994, 1996, and 2000, respectively. From 1999 to 2004, he was an assistant professor and from 2004 to 2006, a senior lecturer at Kyoto University. From 2009 to 2011, he was a chair professor at Ritsumeikan University, Japan. Since 2011, he has been an associate professor at Kyoto University and an adjunct professor at Egypt-Japan University of Science and Technology, Egypt. His research interests are in quantum physics and chemistry in materials science and engineering. (koichi@cpier.kyoto-u. ac.jp) 